\title{
Caracterización de las amplificaciones de onda del suelo blando en Cuenca
}

\section{Characterization of soft ground wave amplifications in Cuenca.}

1 Marcelo Espinoza Cárdenas.

https://orcid.org/0000-0003-1667-3560

Facultad de Ingeniería Civil de la Universidad Católica de Cuenca, Unidad Académica de Ingeniería, Industria y Construcción, Cuenca, Av. de las Américas y General Torres. marceloespinoza00@ hotmail.com

2 Pablo Quinde Martínez.

https://orcid.org/0000-0002-9596-6312

Doctor en Ingeniería Civil- Estructuras, Universidad Nacional Autónoma de México. pablo.quindem@gmail.com

Artículo de Investigación Científica y Tecnológica

Enviado: $14 / 12 / 2021$

Revisado: $28 / 12 / 2021$

Aceptado: 25/01/2022

Publicado:11/02/2022

DOI: $\underline{\text { https://doi.org/10.33262/concienciadigital.v5i1.2067 }}$

Cítese: $\quad$ Espinoza Cárdenas, M., \& Quinde Martínez, P. (2022). Caracterización de las amplificaciones de onda del suelo blando en Cuenca. ConcienciaDigital, 5(1), 188-207. https://doi.org/10.33262/concienciadigital.v5i1.2067

CONCIENCIA DIGITAL, es una Revista Multidisciplinar, Trimestral, que se publicará en soporte electrónico tiene como misión contribuir a la formación de profesionales competentes con visión humanística y crítica que sean capaces de exponer sus resultados investigativos y científicos en la misma medida que se promueva mediante su intervención cambios positivos en la sociedad. https://concienciadigital.org La revista es editada por la Editorial Ciencia Digital (Editorial de prestigio registrada en la Cámara Ecuatoriana de Libro con No de Afiliación 663) www.celibro.org.ec 
claves: ondas

sísmicas,

refracción,

funciones de

transferencia.
Palabras

Introducción. La problemática causada por los suelos blandos dentro de la ciudad de Cuenca, se ve cada vez incrementada debido a la expansión poblacional de la ciudad, especialmente en dos zonas periféricas: Challuabamba y Nulti, presentándose casos de inestabilidad de taludes, trisaduras graves en mamposterías, hundimientos, etc. Objetivo. En el presente artículo, se hace un análisis de como las ondas sísmicas se amplifican o se atenúan en suelos blandos y nos permiten determinar el pico más alto de frecuencias y por ende calcular el periodo fundamental del suelo. Metodología. Para determinar los registros sísmicos se hizo a través de ensayos geofísicos de Refracción Sísmica mediante el método MASW, se tendió seis líneas sísmicas, dos en Challuabamba y cuatro en Nulti, que nos permitieron determinar las velocidades de onda de compresión (Vp), onda de corte (Vs), y varios parámetros elásticos de los suelos estudiados. Resultados. Con los parámetros elásticos calculados, y mediante el programa DEGTRA se realizó una modelación matemática, haciendo uso de las Funciones de Transferencia Teóricas, se obtuvo como resultado la determinación de la máxima frecuencia del suelo y a su vez el periodo del mismo, y realizar una comparación con el periodo fundamental calculado mediante la refracción sísmica. Conclusión. Mediante las Funciones de Transferencia podemos modelar como se amplifican o se atenúan las ondas sísmicas al transmitirse dentro de un estrato de suelo al percibir un movimiento telúrico, que día a día están presentes en nuestra vida cotidiana y en especial en zonas con un alto peligro sísmico, en donde los efectos de sitio son los modeladores de las amplificaciones de onda en suelos blandos.

\section{Keywords:}

seismic waves, refraction, transfer functions.

\section{Abstract}

Introduction. The problem caused by soft soils within the city of Cuenca, is increasingly increased due to the population expansion of the city, especially in two peripheral areas: Challuabamba and Nulti, presenting cases of slope instability, serious cracks in masonry, sinkholes etc. Target. In this article, an analysis is made of how seismic waves are amplified or attenuated in soft soils and allow us to determine the highest peak of frequencies and therefore calculate the fundamental period of the soil. Methodology. To determine the seismic records, it was done through geophysical tests of Seismic Refraction using the MASW method, six seismic lines 


\begin{abstract}
were laid, two in Challuabamba and four in Nulti, which allowed us to determine the compression wave velocities (Vp), shear (Vs), and various elastic parameters of the soils studied. Results. With the elastic parameters calculated, and through the DEGTRA program, a mathematical modeling was carried out, using the Theoretical Transfer Functions, the result was the determination of the maximum frequency of the soil and in turn the period of the same, and make a comparison with the fundamental period calculated by seismic refraction. Conclusion. Through the Transfer Functions we can model how seismic waves are amplified or attenuated when transmitted within a soil stratum when perceiving a telluric movement, which are present day by day in our daily lives and especially in areas with a high seismic hazard., where site effects are the shapers of wave amplifications in soft soils.
\end{abstract}

\title{
Introducción
}

La ocurrencia natural de los terremotos ha probado ser un gran desafío en todas las actividades ligadas al desarrollo socioeconómico de los países. El desconocimiento del potencial de impacto de este tipo de eventos, ha derivado en graves consecuencias en términos de pérdidas económicas y de vidas humanas (Vanegas, 2020).

Debido a su ubicación geográfica, Ecuador se encuentra dentro del cinturón de fuego del Pacifico, como se puede observar en la figura 1, que connota el alto peligro sísmico existente en la zona, debido principalmente a la interacción que se producen entre las placas tectónicas de Nazca y Sudamericana a lo largo de la cordillera de los Andes (Taipe, 2013).

\section{Figura 1}

\section{Distribución de las principales estructuras morfo-tectónicas de los Andes}

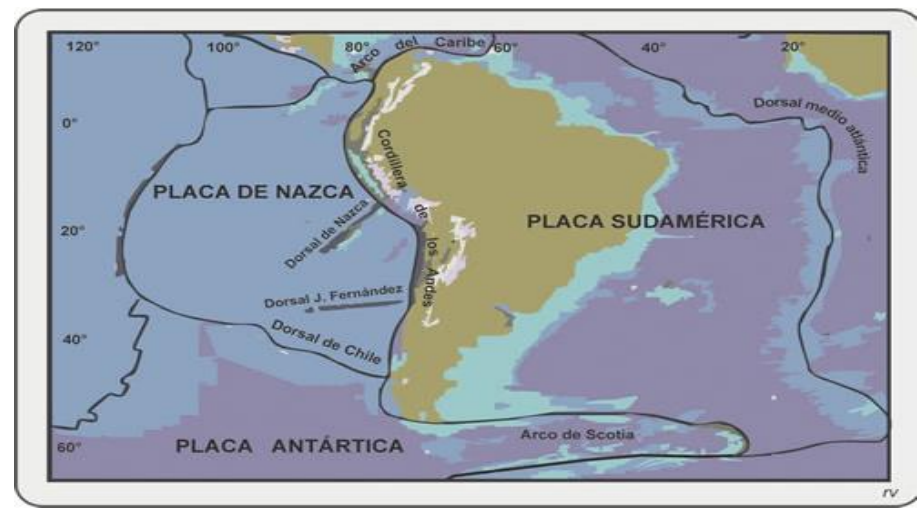

Nota: Adaptado de Manual de Geología, INSUGEO, Misceláneo 21 
Aunque en la ciudad de Cuenca, Ecuador, no se han presentado sismos de alta intensidad en los últimos cien años, la sismicidad histórica recogida en el catálogo elaborado por la Red Sísmica del Austro, da cuenta de la ocurrencia de sismos importantes: en 1758, en febrero de 1856, el 29 de junio de 1887 y el 23 de febrero de 1913, siendo el más severo el sismo de 1887 (Jiménez et al., 2018).

El movimiento generado por un sismo, depende de los parámetros asociados a la fuente sísmica y de las condiciones locales, tales como las irregularidades geológicas, irregularidades topográficas, así como a los efectos locales de sitio que dependen del tipo de suelo y de la amplificación de la onda sísmica que podrían producirse (Vanegas, 2020).

En la actualidad es común analizar los efectos de sitio mediante la estimación de las frecuencias a las cuales se espera amplificación y amplitud de esta, ya sea utilizando pruebas de geofísica (refracción símica) o pruebas de vibración ambiental. Una vez determinada la estructura del subsuelo es posible utilizar simulación numérica de propagación de ondas sísmicas (Funciones de Transferencia) para calcular los efectos de sitio esperados (Chávez, 2014).

En este artículo se presenta un estudio de las características de la amplificación de onda del suelo, mediante una modelación matemática (Funciones de Transferencia), realizadas en dos zonas en expansión urbana de la ciudad de Cuenca, partiendo de las características de una columna de suelo obtenida a través de ensayos de refracción sísmica

\section{Zona de estudio}

El estudio se realizó en la ciudad de Cuenca perteneciente a la provincia del Azuay, Ecuador. Parte de la expansión urbana se ha desarrollado hacia el sector Noreste de la urbe en dos zonas periféricas: Nulti y Challuabamba, situadas a $12 \mathrm{~km}$ del centro urbano, estas zonas presentan un alto índice habitacional, aunque se pueden visualizar problemas de suelos blandos (arcillas expansivas), como son: fallas de hundimientos en cimentaciones, cuarteaduras en paredes, fisuramientos de suelos. Los ensayos de Refracción Sísmica se realizaron en la Parroquia Challuabamba, en dos sub zonas mostradas en la figura 2, Colinas de Challuabamba Alta y en la Autopista CuencaAzogues a la altura del Puente Sixto Durán Ballén, realizándose en cada una de ellas un ensayo de sísmica de refracción, y se contó con los registros sísmicos de cuatro líneas sísmicas tendidas en la parroquia Nulti. 


\section{Figura 2}

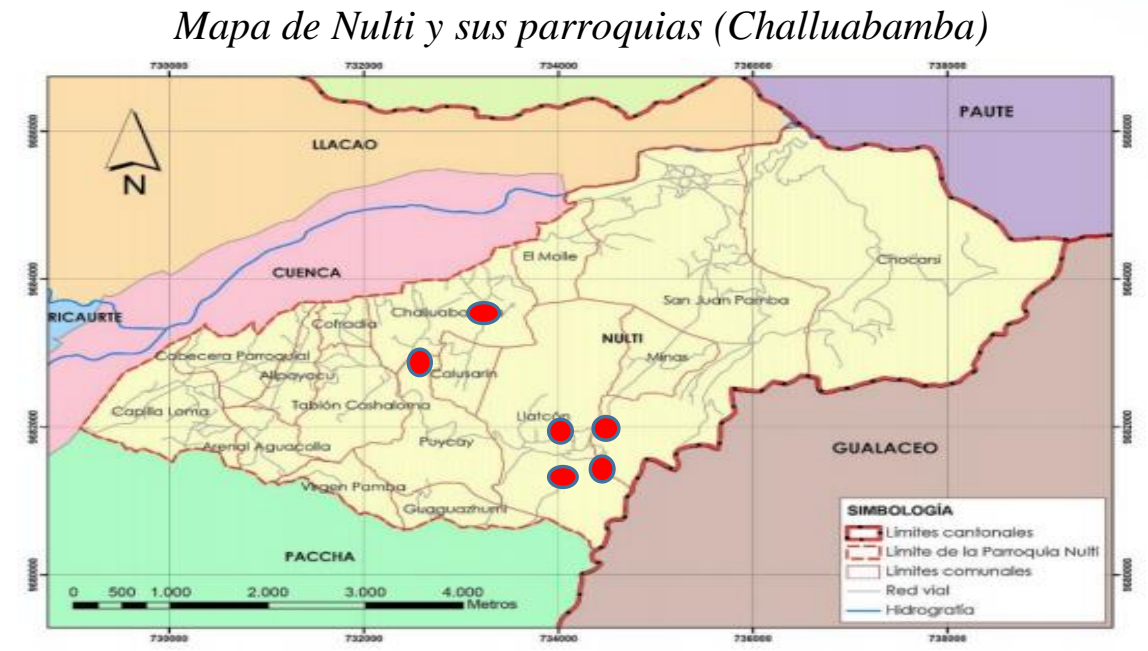

Fuente: Proyecto Prevención de Desastres Naturales de la Cuenca del Paute Geología de la Zona

En función de la información expuesta en el Proyecto PRECUPA (Proyecto Prevención de Desastres Naturales de la Cuenca del Paute), se evidencia que en las zonas de Nulti y Challuabamba existen dos unidades geológicas dominantes: Depósitos Coluviales (Qc) y la Formación Loyola (ML).

\section{Depósitos Coluviales $(Q c)$}

Depósitos de ladera y de pie de talud con materiales que han sufrido poco transporte, son muy heterogéneos dependiendo del aporte y se pueden originar por varias formas como: deslizamientos, derrumbes, flujos, entre otros. De manera general se componen de mezclas heterogéneas de bloques y fragmentos angulares - sub angulares en matriz limo arcillosa o arenosa. En la zona de estudio los depósitos coluviales están asociados a escarpes activos latentes y relictos (ver Figura 3).

\section{Figura 3}

\section{Deposito Coluvial Sector de Challuabamba}

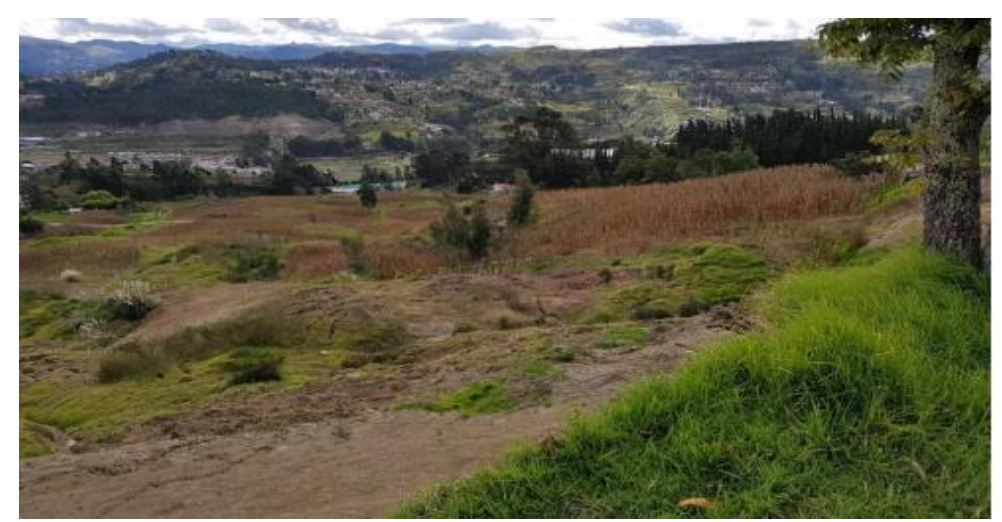

Nota: Adoptado de Estudio Geológico y Geotécnico de Challuabamba, Gestión de Riesgos Municipio de Cuenca 


\section{Formación Loyola $(M L)$}

Formación sedimentaria clástica fino granular, como se puede apreciar en la Figura No.4, consiste en una alternancia de lutitas gris oscuro muy fisibles, limolitas que meteorizan a arcillas de color blanco y habano, localmente lentes de areniscas limosas habanas finamente estratificadas con cemento calcáreo, calizas, y mega brechas. En el lado oriental de la cuenca, en la base de la formación ocurren areniscas y conglomerados (básales) en discordancia sobre la Formación Yunguilla. Abunda yeso, especialmente en las superficies alteradas.

\section{Figura 4}

Formación Loyola

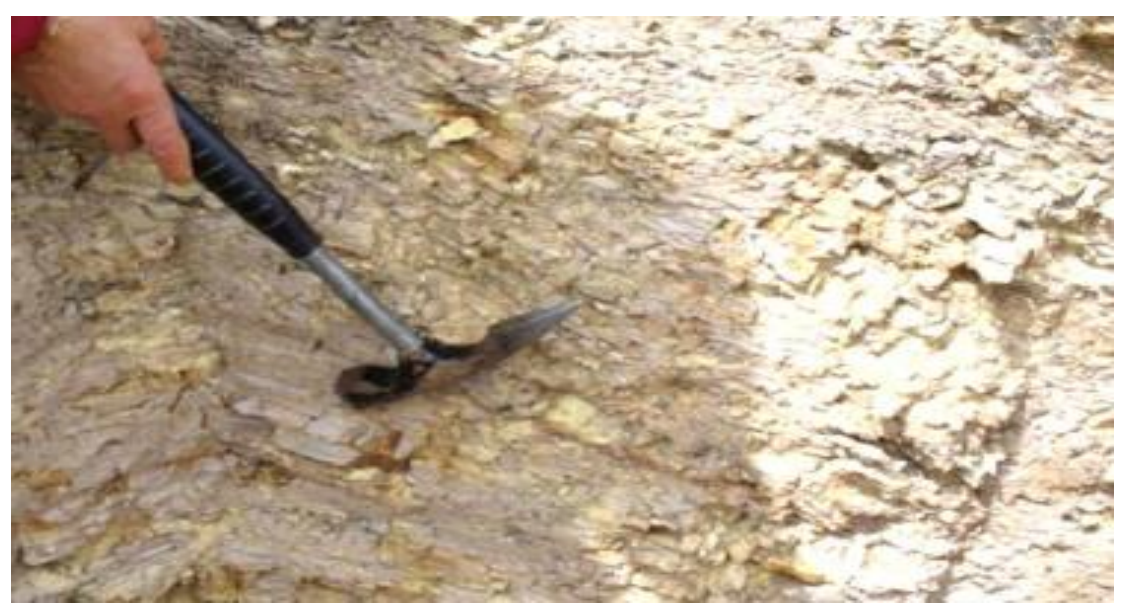

Nota: Proyecto Prevención de Desastres Naturales de la Cuenca del Paute

\section{Metodología}

\section{Ensayo de Refracción Sísmica}

El ensayo, consiste en registrar los tiempos de viaje de las ondas sísmicas hasta una serie de geófonos superficiales, instalados en una línea recta con origen en el punto de emisión. En casos de prospecciones profundas, es común el uso de explosivos, con lo cual la mayor fracción de la energía se transmite en ondas de compresión. Cuando las prospecciones son poco profundas, se puede utilizar herramientas manuales, para transmitir ondas de un modo que en una dirección determinada se propaguen las mismas siendo fundamentalmente de compresión o de corte (Ampuero \& Van Sint, 2004).

\section{Equipo Utilizado}

Al llevar a cabo el ensayo de sísmica de refracción se utilizó el siguiente equipo:

- Sismógrafo PASI Gea24.

- Geófonos verticales de $4.5 \mathrm{~Hz}$. 
- Martillo de 16 libras.

\section{Figura 5}

Ensayo de Refracción Sísmica en Challuabamba

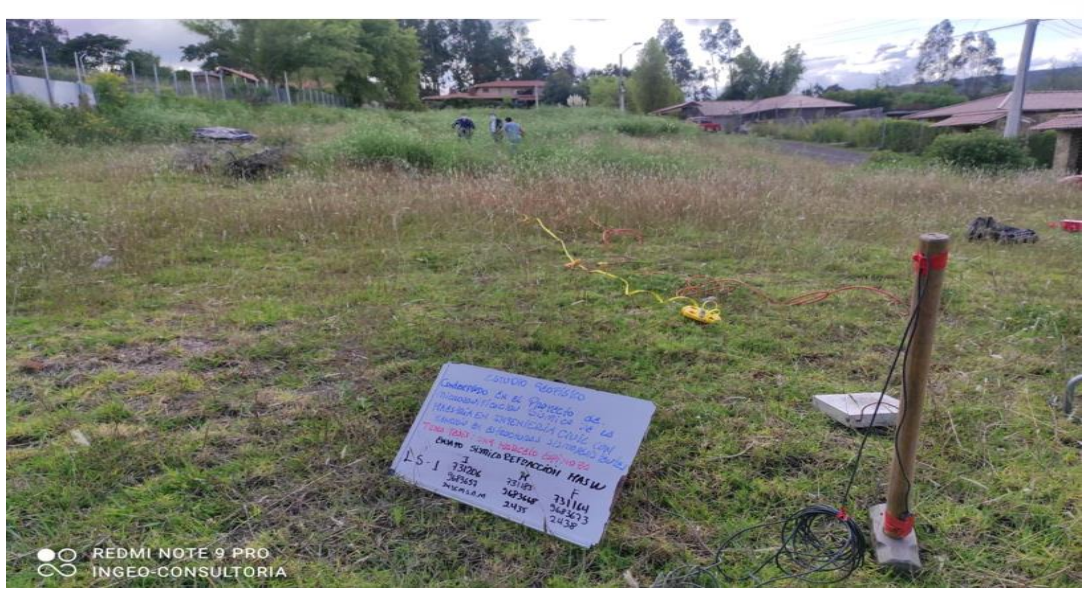

Se realizó el ensayo con el tendido de dos líneas sísmicas en el área de Challuabamba, y además se tomó los resultados de cuatro líneas sísmicas en el sector de Nulti, realizados por el laboratorio de suelos de la Universidad Católica de Cuenca (UCACUE) en un estudio realizado en mayo del 2021.

Cada tendido de la línea de refracción tiene una longitud de escaneo de 50 metros, se colocó 24 geófonos separados $2 \mathrm{~m}$ entre ellos y mediante un martillo de 16 libras se generó el impacto sobre una placa de metal transmisora de ondas hacia los geófonos, los resultados del tiempo de registro de las distintas ondas sísmicas se registran en el sismógrafo y mediante el método Análisis Multicanal de Ondas Superficiales (MASW), fue posible determinar un perfil de Velocidades de Onda.

El método (MASW) permite conocer el perfil de velocidad de las ondas de corte (Vs) en el subsuelo en base a la propiedad de dispersión de las ondas superficiales (velocidad de fase). Luego, a través de un proceso de inversión no lineal, se obtiene el perfil teórico que se ajuste a la curva de dispersión experimental (figura 7). Para el análisis de los resultados se considera la clasificación que la Norma Ecuatoriana de la Construcción 2015, Diseño Sismo Resistente, establece para las velocidades de las ondas de corte (Vs). 


\section{Figura 6}

Grafica de Velocidades de Onda Vp y Vs $(\mathrm{m} / \mathrm{seg})$

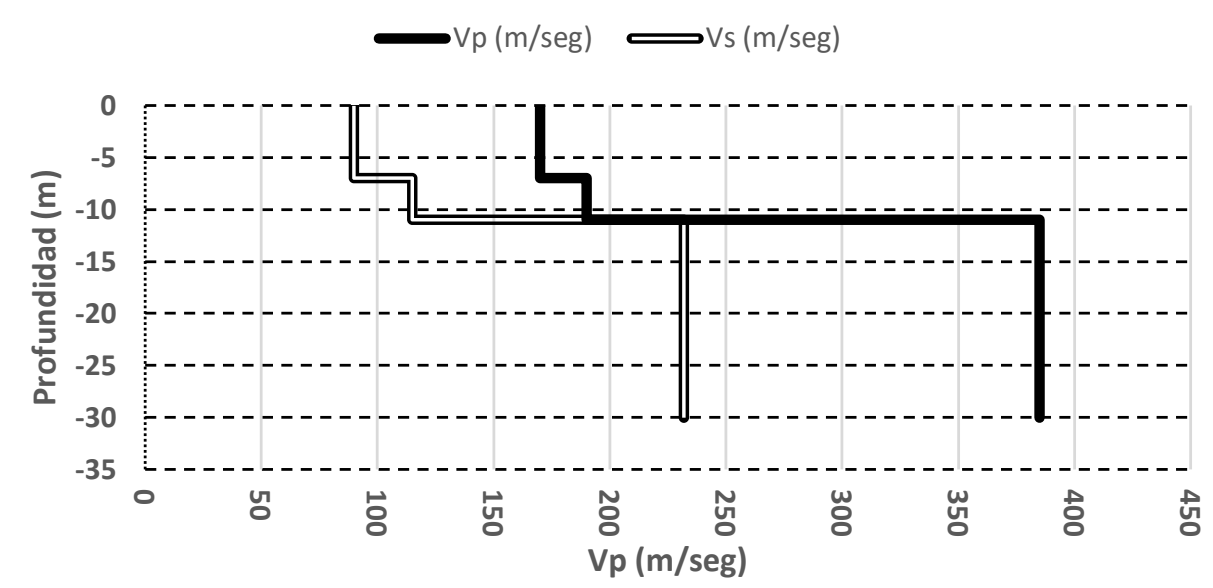

\section{Modelación Matemática}

Se realizó una modelación matemática de las amplificaciones o atenuaciones de onda, mediante las Funciones de Transferencia Teóricas, que nos permitieron obtener la amplificación de las ondas del suelo en función de su estratigrafía. Una Función de Transferencia se la puede definir como la relación existente entre la Transformada Rápida de Fourier del registro de aceleración en superficie y la Transformada de Fourier del registro de aceleración en roca, de esta manera la Función de Transferencia determina como cada frecuencia en el movimiento en la roca es amplificado o atenuado por el depósito de suelo.

Para determinar la respuesta dinámica del terreno ante un sismo, es importante conocer la influencia de algunas propiedades, tales como la velocidad de onda de corte (Vs), el espesor de la capa de suelo $(\mathrm{H})$ y la densidad $(\rho)$ de cada capa de subsuelo, como también las características del movimiento de entrada (registro de aceleración en roca).

Este tipo de análisis sirve de apoyo en trabajos en donde queremos dividir una zona en micro zonas con características dinámicas semejantes ante la presencia de sismos, a lo que se le conoce como microzonificación sísmica. Con este tipo de estudios podemos conocer los parámetros dinámicos y así realizar un modelamiento de la respuesta sísmica del suelo, lo cual nos permite identificar cómo será la respuesta del suelo ante cualquier sismo que se registre en la zona.

Para el modelado de las Funciones de Transferencia se utilizó el programa DEGTRA, desarrollado por un grupo de investigadores y estudiantes del Instituto de Ingeniería de la Universidad Nacional Autónoma de México, (UNAM). DEGTRA nos permite la evaluación de funciones de transferencias (FT) a partir de espectros de Fourier y permite realizar cálculos de funciones de transferencia teóricas con el método de Thomson 
Haskell, dicho método se basa en un modelo en capas, comúnmente utilizado en geofísica para analizar el comportamiento de las ondas en diferentes estratos, para obtener las frecuencias de vibración del suelo (Álvarez-Icaza, 2014).

La idea de este método consiste en detectar cambios en el tiempo de llegada de las ondas que se propagan a través del suelo y, con ello identificar las frecuencias de vibración de esta, entre ellas la frecuencia fundamental. Esta técnica tiene la ventaja de identificar un número relativamente pequeño de parámetros. Los resultados de simulación muestran como estos son consistentes con los tiempos de viaje de onda esperados y se concluye que la técnica propuesta es prometedora para identificación y localización de cambios en su frecuencia natural (Álvarez-Icaza. 2014).

Mediante una señal de entrada, e(t), generada por el sismo, que al pasar por la masa de suelo sufre una transformación, $\mathrm{H}$, y genera una señal de salida, $\mathrm{s}(\mathrm{t})$, que produce el movimiento del suelo en superficie, el método trabaja asumiendo un comportamiento lineal del suelo (Vanegas, 2020).

\section{Ecuación 1.}

$$
s(t)=H[e(t)]
$$

En nuestro caso, a partir de los resultados obtenidos a través de los ensayos de Refracción Sísmica de seis puntos analizados, se obtuvo las Funciones de Transferencia Teóricas, en las que según sus amplificaciones de onda máximos corresponderán a las mayores frecuencias naturales, mediante las cuales y calculando sus valores inversos se determinará el periodo fundamental del suelo.

\section{Resultados}

\section{Parámetros Elásticos determinados con Refracción Sísmica}

Una de las ventajas que presentan los ensayos geofísicos de sísmica de refracción, es que se puede determinar una serie de parámetros elásticos de los materiales del subsuelo; Los módulos elásticos del suelo son útiles para una variedad de aplicaciones dentro de la ingeniería civil, especialmente en el área de geotecnia.

Las principales aplicaciones de los módulos elásticos son el cálculo de asentamientos y deformaciones en el suelo y la determinación de la compresibilidad del suelo. A continuación, en la tabla 1, se muestran varios parámetros elásticos, inclusive el periodo fundamental del suelo, en función de conocer las ondas compresionales y cortantes. 
Tabla 1

Parámetros elásticos determinados mediante refracción sísmica

\begin{tabular}{|c|c|c|c|c|c|c|c|c|c|c|c|c|c|}
\hline UBICACIÓN & $\begin{array}{c}\# \\
\text { Capas }\end{array}$ & $\begin{array}{l}\text { Profundid } \\
\text { ad (m) }\end{array}$ & $\underset{(\mathbf{m} / \mathbf{s e g})}{\mathbf{V p}}$ & $\begin{array}{c}\begin{array}{c}\text { Vs } \\
(\mathbf{m} / \mathbf{s e g})\end{array} \\
.\end{array}$ & $\begin{array}{c}\text { P. } \\
\text { Especifico } \\
(\mathrm{Kn} / \mathrm{m} 3)\end{array}$ & $\begin{array}{c}\text { C. } \\
\text { Admisible } \\
(\mathrm{Kn} / \mathrm{m} 2)\end{array}$ & $\begin{array}{c}\text { Módulo } \\
\text { de Corte } \\
(\mathrm{Kn} / \mathrm{m} 2)\end{array}$ & $\begin{array}{c}\text { C. } \\
\text { Poisson }\end{array}$ & $\begin{array}{c}\text { M. Young } \\
\text { (Kn/m2) }\end{array}$ & $\begin{array}{c}\text { M. } \\
\text { Endomet } \\
\text { rico } \\
(\mathbf{K n} / \mathbf{m} 2)\end{array}$ & $\begin{array}{l}\text { M. Bulk } \\
\text { (kn/m2) }\end{array}$ & $\begin{array}{c}\text { M. } \\
\text { Balasto } \\
(\mathrm{kn} / \mathrm{m} 2)\end{array}$ & $\begin{array}{l}\text { Periodo } \\
\text { (seg) }\end{array}$ \\
\hline C. de Challuabamba & 1 & 10.3 & 193 & 117 & 1193 & 34.89 & 16643.4 & 0.21 & 40259.93 & 13156.83 & 23097 & 52634.8 & 0.35 \\
\hline C. de Challuabamba & 2 & 19.7 & 384 & 232 & 1417 & 82.16 & 77721.24 & 0.21 & 188486 & 61199.5 & 109296 & 246758 & 0.34 \\
\hline Puente Sixto Duran & 1 & 16 & 225 & 134 & 12.39 & 41.52 & 22684.86 & 0.23 & 55586.22 & 17576.62 & 33711 & 73194.3 & 0.48 \\
\hline UBICACIÓN & $\begin{array}{c}\# \\
\text { Capas }\end{array}$ & $\begin{array}{l}\text { Profundid } \\
\text { ad (m) }\end{array}$ & $\begin{array}{c}\mathbf{V p} \\
(\mathbf{m} / \mathbf{s e g})\end{array}$ & $\begin{array}{c}\mathbf{V s} \\
(\mathbf{m} / \mathbf{s e g})\end{array}$ & $\begin{array}{c}P . \\
\begin{array}{c}P \text { Especifico } \\
(\mathbf{K n} / \mathbf{m} 3)\end{array} \\
\end{array}$ & $\begin{array}{c}\text { C. } \\
\text { Admisible } \\
(\mathbf{K n} / \mathbf{m} 2)\end{array}$ & $\begin{array}{c}\begin{array}{c}\text { Módulo } \\
\text { de Corte } \\
(\mathbf{K n} / \mathbf{m} 2)\end{array} \\
\end{array}$ & $\begin{array}{c}\text { C. } \\
\text { Poisson }\end{array}$ & $\begin{array}{c}\text { M. Young } \\
\text { (Kn/m2) }\end{array}$ & \begin{tabular}{|c|} 
M. \\
Endomet \\
rico \\
$(\mathrm{Kn} / \mathrm{m} 2)$ \\
\end{tabular} & $\begin{array}{l}\text { M. Bulk } \\
\text { (kn/m2) }\end{array}$ & $\begin{array}{c}\text { M. } \\
\text { Balasto } \\
(\mathbf{k n} / \mathbf{m} 2)\end{array}$ & $\begin{array}{l}\text { Periodo } \\
\text { (seg) }\end{array}$ \\
\hline Puente Sixto Duran & 2 & 14 & 330 & 180 & 13.64 & 61.37 & 45045.82 & 0.29 & 116059.2 & 32062.02 & 91342.9 & 158219 & 0.31 \\
\hline Nulti LS-1 & 1 & 1.5 & 280 & 172 & 13.09 & 56.29 & 39475.48 & 0.2 & 94503.07 & 31699.42 & 51979.3 & 32772.7 & 0.6 \\
\hline Nulti LS-1 & 2 & 28.5 & 315 & 190 & 13.48 & 64.04 & 49609.61 & 0.21 & 120458.03 & 38990.21 & 70211.6 & 42080.9 & 0.6 \\
\hline Nulti LS-2 & 1 & 1.5 & 238 & 146 & 12.57 & 45.88 & 27310.58 & 0.2 & 65453.24 & 21894.54 & 36159.6 & 22710.9 & 0.71 \\
\hline Nulti LS-2 & 2 & 28.5 & 260 & 160 & 12.85 & 51.4 & 33532.37 & 0.2 & 80158.34 & 26985.58 & 43836.6 & 27778.3 & 0.71 \\
\hline Nulti LS-3 & 1 & 1.5 & 250 & 152 & 12.72 & 48.35 & 29967.68 & 0.21 & 73328.33 & 23771.2 & 41110.4 & 25186.3 & 0.7 \\
\hline Nulti LS-3 & 2 & 28.5 & 270 & 164 & 12.97 & 53.18 & 35563.92 & 0.21 & 85899.5 & 28178.09 & 48975.3 & 29923.8 & 0.7 \\
\hline Nulti LS-4 & 1 & 1.5 & 258 & 156 & 12.82 & 50.02 & 31815.23 & 0.21 & 77110.58 & 25075.18 & 44600.9 & 26911.3 & 0.7 \\
\hline Nulti LS-4 & 2 & 28.5 & 270 & 164 & 12.97 & 53.18 & 35563.92 & 0.21 & 85899.5 & 28178.09 & 48975.3 & 29923.8 & 0.7 \\
\hline
\end{tabular}

Entre los principales parámetros elásticos que se pudo determinar con el ensayo de refracción sísmica visualizados en la tabla 1 fueron los siguientes:

También conocido como la capacidad portante o la capacidad que tiene un suelo para soportar presiones verticales, técnicamente la capacidad portante es la máxima presión media de contacto entre la cimentación y el terreno tal que no se produzcan un fallo por cortante del suelo o un asentamiento diferencial excesivo. Es de vital importancia al momento de diseñar una cimentación o para calcular asentamientos del suelo.

Se calculó utilizando la fórmula:

\section{Ecuación 2}

$$
q_{a}=\frac{\gamma}{n} * V s * T
$$

En donde:

$$
\begin{aligned}
& q_{a}=\text { Capacidad admisible. } \\
& \gamma=\text { Peso Especifico del suelo. } \\
& \text { Vs }=\text { Velocidad de onda de corte. } \\
& \mathrm{T}=\text { Tiempo. }
\end{aligned}
$$

\section{Módulo de Corte $(\mathrm{Kn} / \mathrm{m} 2)$}

El módulo de corte $\mathrm{G}$, también denominado módulo de rigidez, es una constante elástica que caracteriza el cambio de forma que experimenta un material elástico (lineal e 
isótropo) cuando se aplican esfuerzos cortantes. Describe el cizallamiento o cambio de fuerza del suelo (Mohammed et al., 2020).

Se calculó utilizando la fórmula:

\section{Ecuación 3}

$$
G=\frac{\gamma}{g} * V s^{2}
$$

En donde:

$$
\begin{aligned}
& \mathrm{G}=\text { Modulo de Corte }(\mathrm{Kn} / \mathrm{m} 2) \\
& \gamma=\text { Peso Especifico del suelo } \\
& \text { Vs }=\text { Velocidad de onda de corte. }
\end{aligned}
$$

\section{Coeficiente de Poisson}

$\mathrm{Al}$ aplicarse una fuerza sobre un cuerpo, este presenta una deformación transversal, cuya dirección depende del sentido de la aplicación de la fuerza axial. Cuando la fuerza axial a la que está sometido el material es de compresión, en el sentido axial se experimentará una contracción, mientras que en el sentido transversal existirá una expansión del material, en cambio, si la fuerza axial es de tensión en el sentido axial se experimentará una expansión y en el sentido transversal habrá una contracción (Negreros \& González, 2018).

Se calculó utilizando la fórmula:

\section{Ecuación 4}

$$
\boldsymbol{\mu}_{\boldsymbol{d}}=\frac{(V p / V s)^{2}-2}{2 *\left(\frac{V p}{V s}\right)^{2}-2}
$$

En donde:

$$
\begin{aligned}
& \mu_{\mathrm{d}}=\text { Coeficiente de Poisson. } \\
& \mathrm{Vp}=\text { Velocidad de onda longitudinal. } \\
& \mathrm{Vs}=\text { Velocidad de onda de corte. }
\end{aligned}
$$




\section{Módulo de Young (Kn/m2)}

El módulo de elasticidad (E), también conocido como el módulo de Young, es la relación que existe entre un esfuerzo axial y la deformación unitaria que presenta un material sometido a este esfuerzo dentro del rango elástico de la curva esfuerzo-deformación, es decir hasta el límite elástico del material (Negreros \& González, 2018).

Se calculó utilizando la fórmula:

\section{Ecuación 5}

$$
\boldsymbol{E}=\delta * V s^{2} * \frac{\left(3 * V p^{2}-4 * V s^{2}\right)}{\left(V p^{2}-V s^{2}\right)}
$$

En donde:

$$
\begin{aligned}
& \mathrm{E}=\text { Modulo de Young } \\
& \delta=\text { Densidad del Suelo } \\
& \mathrm{Vp}=\text { Velocidad de onda longitudinal. } \\
& \mathrm{Vs}=\text { Velocidad de onda de corte. }
\end{aligned}
$$

\section{Módulo Edometrico $(\mathrm{Kn} / \mathrm{m} 2)$}

Se trata de un parámetro similar al módulo de elasticidad, pero en este caso el módulo edometrico de deformación, es un módulo de compresión confinada sin deformaciones horizontales y solo tiene validez para determinados valores de carga (Ilori, 2019).

Se calculó utilizando la fórmula:

\section{Ecuación 6}

$$
\text { Eoed }=\frac{\left(1-\mu_{d}\right) * E}{\left(1-2 * \mu_{d}\right) *\left(1+\mu_{d}\right)}
$$

En donde:

$$
\begin{aligned}
& \text { Eoed = Módulo Edométrico. } \\
& \text { E = Módulo Elástico. } \\
& \mu_{\mathrm{d}}=\text { Coeficiente de Poisson. }
\end{aligned}
$$




\section{Módulo de Bulk (Kn/m2)}

Este parámetro elástico del suelo mide la relación entre una presión hidrostática aplicada a un material y el cambio de volumen presentado por el material. Este módulo indica la resistencia de un material al cambio de su volumen, cuando le son aplicadas cargas distribuidas en todas las caras o lados del material (Ilori, 2019).

Se calculó utilizando la fórmula:

\section{Ecuación 7}

$$
\boldsymbol{K}=\frac{E}{3 *\left(1-2 * \mu_{d}\right)}
$$

En donde:

$$
\begin{aligned}
& \mathrm{K}=\text { Módulo de Bulk. } \\
& \mathrm{E}=\text { Módulo de Young. } \\
& \mu_{\mathrm{d}}=\text { Coeficiente de Poisson. }
\end{aligned}
$$

\section{Módulo de Balasto (Kn/m2)}

El módulo de balasto es una magnitud asociada a la rigidez del terreno. Permite conocer el asentamiento de una edificación en el terreno, así como la distribución de esfuerzos en ciertos elementos de cimentación. Se mide aplicando una carga vertical sobre una superficie y midiendo el hundimiento o desplazamiento a partir de la carga aplicada (Mohammed et al., 2020).

Se calculó utilizando la fórmula:

\section{Periodo Fundamental de Vibración Ambiental (seg)}

Es uno de los indicadores más representativos de los efectos dinámicos de sitio de un suelo, representa el tiempo que se demora una onda sísmica en cumplir un ciclo, es de vital importancia para el diseño de estructuras de cimentación y edificaciones (Pickering, 1970).

Se calculó utilizando la fórmula.

\section{Ecuación 8}

$$
\boldsymbol{T}_{s}=\frac{4 * H_{s}}{V_{s}}
$$


En donde:

Ts: Periodo Fundamental del Estrato.

Hs: Espesor total del Estrato.

Vs: Velocidad de corte.

\section{Discusión}

Según los datos observados en la tabla 1, mediante los ensayos de refracción sísmica, realizados en seis líneas sísmicas obtuvimos en cada uno de los ensayos dos capas de suelo con profundidades variables y con velocidades de corte que varían desde los 117 $\mathrm{m} / \mathrm{s}$ hasta los $232 \mathrm{~m} / \mathrm{s}$, además se puede observar para el sector de Challuabamba un valor promedio de 0,37 segundos para el Periodo Fundamental y para la zona de Nulti de 0,68 segundos.

En Cuenca, de manera general la mayoría de suelos se clasifican entre tipo B con periodos fundamentales que varían entre 0,1 y 0,25 segundos, y suelos tipo $\mathrm{C}$ con periodos fundamentales que varían entre 0,1 y 0,50 segundos (Bermeo, 2017).

\section{Perfil del suelo en Función de la Velocidad de Corte (Vs).}

Para poder determinar el tipo de perfil en función del tipo de suelo, es necesario determinar la velocidad de onda cortante Vs, que se obtuvo de los ensayos de prospección geofísica de sísmica de refracción, principalmente del ensayo MASW.

A continuación, visualizamos la tabla 2 con la clasificación del suelo en función de las velocidades de corte Vs (m/seg) según lo establecido por la Norma Ecuatoriana de la Construcción.

\section{Tabla 2}

Clasificación del suelo en función de las velocidades de corte

\begin{tabular}{clc}
\hline Tipo de Perfil & \multicolumn{1}{c}{ Descripción } & Definición \\
\hline A & Perfil de roca competente & Vs $>1500 \mathrm{~m} / \mathrm{s}$ \\
B & Perfil de roca de rigidez media & $1500 \mathrm{~m} / \mathrm{s}<\mathrm{Vs}>760 \mathrm{~m} / \mathrm{s}$ \\
C & $\begin{array}{l}\text { Perfiles de suelos muy densos o roca blanda, que } \\
\text { cumplan con el criterio de velocidad de la onda de } \\
\text { cortante }\end{array}$ & $760 \mathrm{~m} / \mathrm{s}<\mathrm{Vs}>360 \mathrm{~m} / \mathrm{s}$ \\
& $\begin{array}{l}\text { Perfiles de suelos muy densos o roca blanda que } \\
\text { cumplan con cualquiera de los dos criterios }\end{array}$ & $\mathrm{Nu}>100 \mathrm{kpa}$
\end{tabular}




\section{Tabla 2}

Clasificación del suelo en función de las velocidades de corte (continuación)

\begin{tabular}{|c|c|c|}
\hline Tipo de Perfil & Descripción & Definición \\
\hline \multirow[t]{2}{*}{$\mathrm{D}$} & $\begin{array}{l}\text { Perfiles de suelos rígidos que cumplan con el criterio de } \\
\text { velocidad de onda de cortante }\end{array}$ & $360 \mathrm{~m} / \mathrm{s}>\mathrm{Vs} \geq 180 \mathrm{~m} / \mathrm{s}$ \\
\hline & $\begin{array}{l}\text { Perfiles de suelos rígidos que cumplan cualquiera de las } \\
\text { dos condiciones }\end{array}$ & $\frac{50,0>\mathrm{N} \geq 15,0}{100 \mathrm{Kpa}>\mathrm{Su} \geq 50 \mathrm{Kpa}}$ \\
\hline \multirow[t]{2}{*}{$\mathrm{E}$} & Perfil que cumpla el criterio de velocidad cortante & $\mathrm{Vs}<180 \mathrm{~m} / \mathrm{s}$ \\
\hline & $\begin{array}{l}\text { Perfil que contiene un espesor total } \mathrm{H} \text { mayor de } 3 \mathrm{~m} \text { de } \\
\text { arcillas blandas }\end{array}$ & $\begin{aligned} \mathrm{W}>40 \% \\
\mathrm{Su}<50 \mathrm{Kpa}\end{aligned}$ \\
\hline $\mathrm{F}$ & $\begin{array}{l}\text { Perfiles de suelo tipo F que requieran una evaluación } \\
\text { realizada explícitamente en el } \\
\text { sitio por un ingeniero geotecnista }\end{array}$ & \\
\hline
\end{tabular}

Fuente: Norma Ecuatoriana de la Construcción 2015, diseño sismo resistente

Al comparar las velocidades de corte Vs, determinadas con los ensayos de refracción sísmica, con la tabla 2 de perfiles de suelo, podemos concluir que al obtener velocidades de corte menores a $230 \mathrm{~m} / \mathrm{s}$, se puede inferir que los suelos analizados pertenecen a tipos de suelo entre D y E, común entre estos suelos: ser blandos y arcillosos.

\section{Modelación matemática mediante las Funciones de Transferencia (FT)}

Luego de ser ingresados al programa Degtra, los resultados obtenidos de los ensayos de refracción sísmica, mediante capas indicando su profundidad $(\mathrm{H})$, la densidad del suelo $(\rho)$, la velocidad de corte (Vs) y un coeficiente de amortiguamiento (\%). Las Funciones de transferencia obtenidas se muestran en las figuras 7 a 12 :

\section{Figura 7}

Resultados de las funciones de transferencia muestra 1

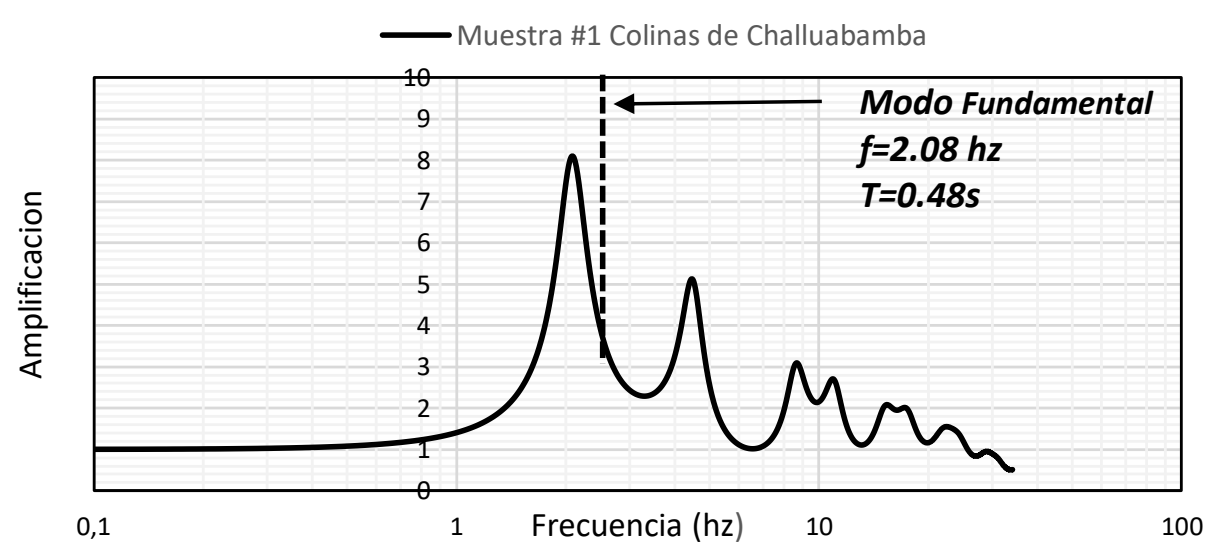




\section{DDigital}

\section{Figura 8}

Resultados de las funciones de transferencia muestra 2

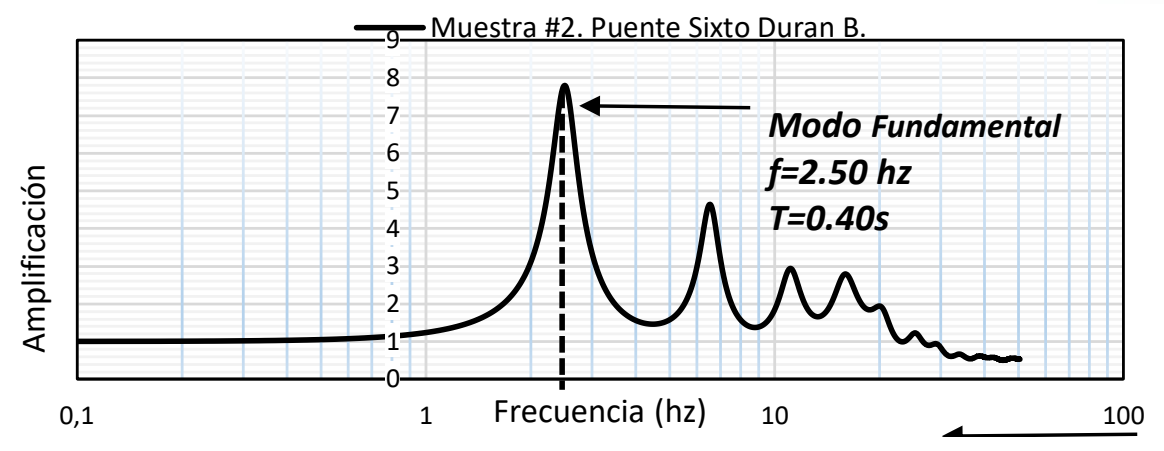

Figura 9

Resultados de las funciones de transferencia línea sísmica 1

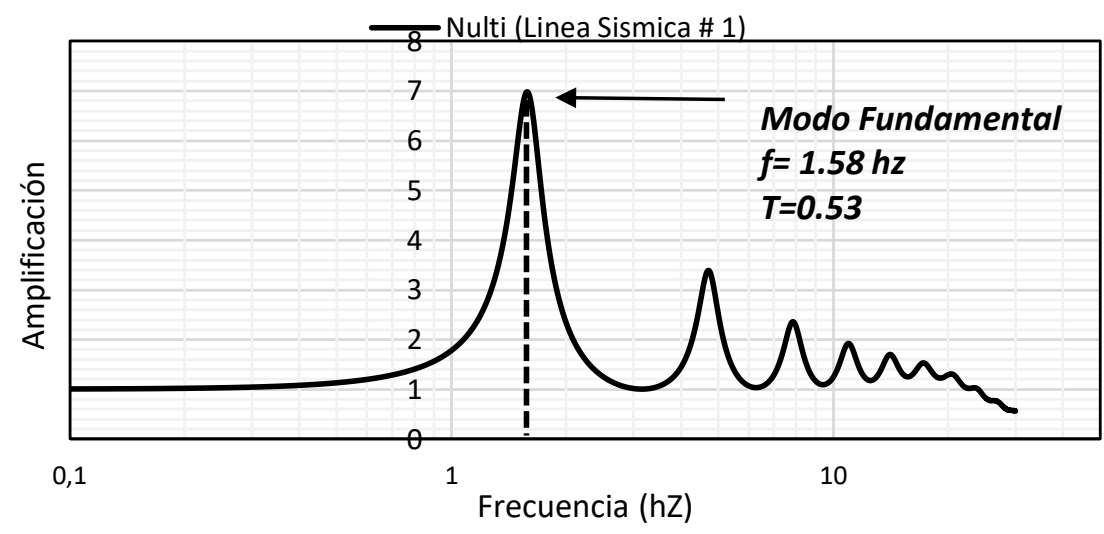

Figura 10

Resultados de las funciones de transferencia línea sísmica 2

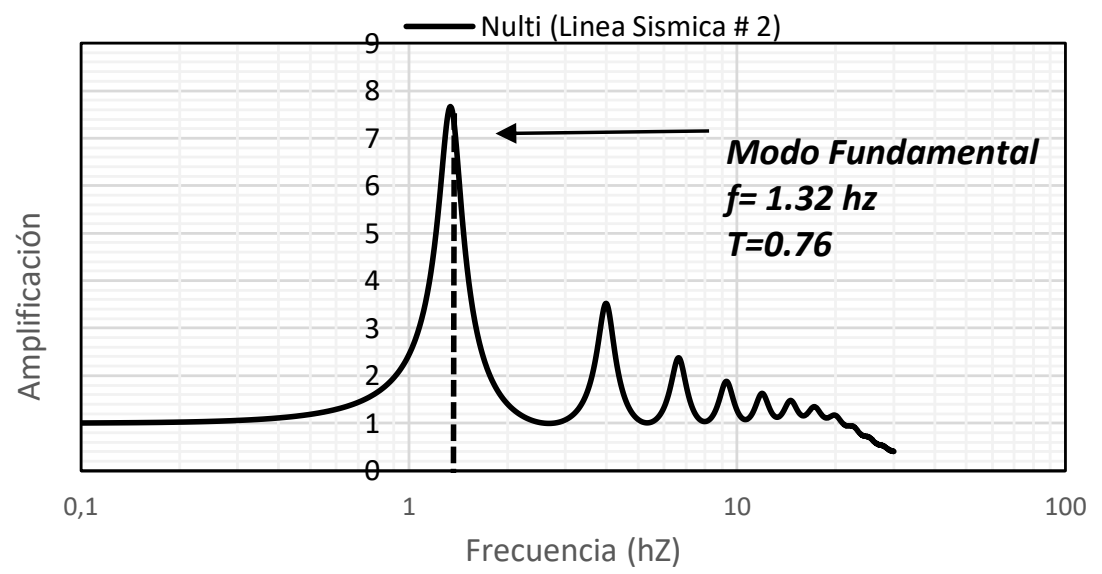




\section{Conciencia}

\section{Figura 11}

Resultados de las funciones de transferencia línea sísmica 3

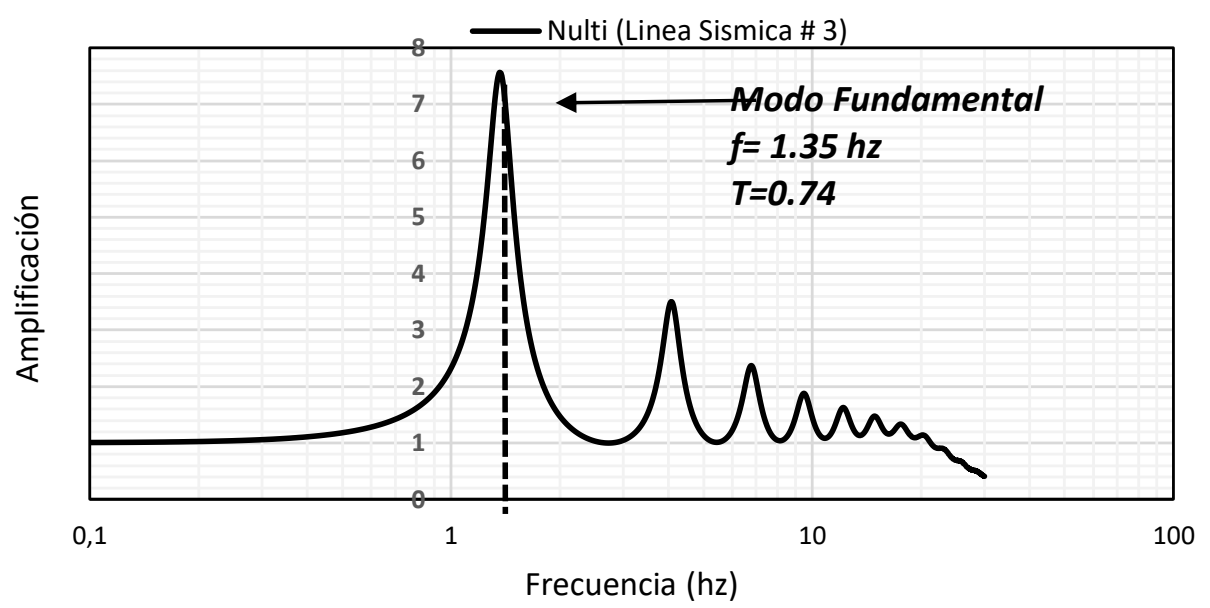

Figura 12

Resultados de las funciones de transferencia línea sísmica 4

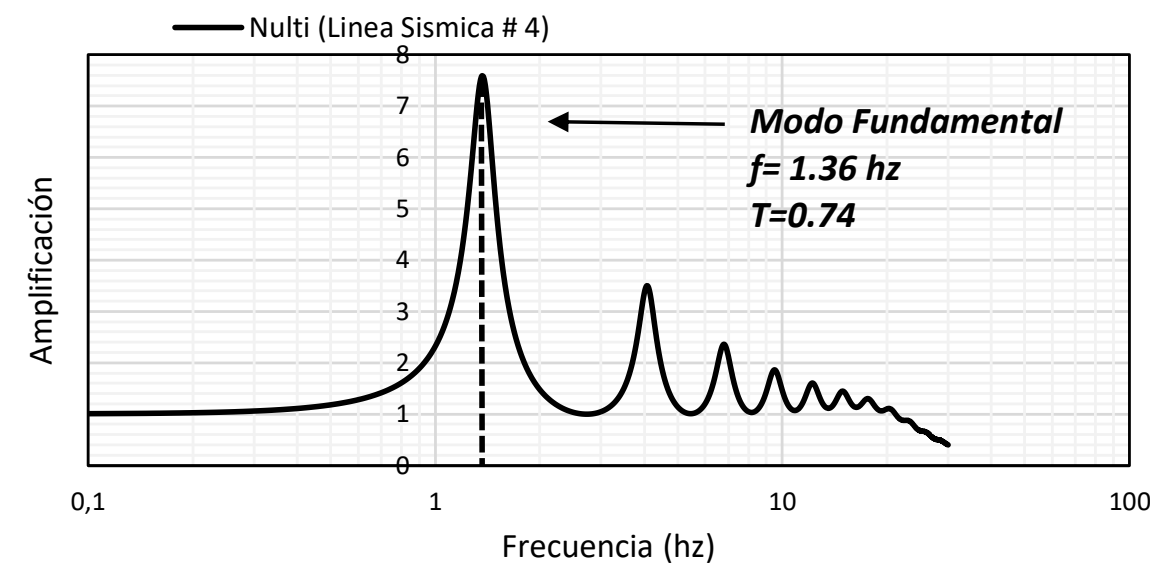

\section{Conclusiones}

Como característica fundamental de las funciones de transferencia teóricas determinadas en este artículo, es la amplificación de la onda dentro de un rango común de frecuencia para todos los gráficos. Se puede observar que las amplificaciones de onda de las FT para la zona de Challuabamba en las figuras 7 y 8 están dentro de un rango de frecuencias 
entre 2 y $2.5 \mathrm{~Hz}$, y para la zona de Nulti en un rango de frecuencias entre 1.3 y $1.6 \mathrm{~Hz}$ en las figuras $9,10,11$ y 12 .

\section{Tabla 3}

Cuadro comparativo de periodos fundamentales

\begin{tabular}{lcc}
\hline \multirow{2}{*}{ Muestra } & Refracción Sísmica & Función de transferencia \\
\cline { 2 - 3 } & Periodo T (seg) & Periodo T (seg) \\
\hline C. Challuabamba & 0.35 & 0.48 \\
P. Sixto Duran & 0.39 & 0.4 \\
Nulti Ls-1 & 0.6 & 0.63 \\
Nulti Ls-2 & 0.71 & 0.76 \\
Nulti Ls-3 & 0.7 & 0.74 \\
Nulti Ls-4 & 0.7 & 0.74 \\
\hline
\end{tabular}

Sabiendo que el periodo fundamental del suelo se calcula como el inverso de la frecuencia natural podemos concluir que, mediante el modelamiento de las Funciones de Transferencia Teóricas, determinamos un periodo fundamental para la zona de Challuabamba promedio de 0,44 segundos, y para el sector de Nulti un periodo fundamental de 0,74 segundos.

Estos periodos determinados mediante el modelamiento de las FT mantienen una semejanza con los periodos determinados mediante los ensayos de refracción sísmica detallados en la tabla 1, dicha semejanza corrobora que se tratan de suelos blandos tipo arcillosos limosos característicos de la Formación Loyola.

Queda pendiente, para futuros estudios mediante ensayos de vibración ambiental complementarios a los ensayos realizados dentro de las zonas estudiadas, determinar zonas con características dinámicas semejantes y armar conjuntamente con estudios realizados en otras parroquias una microzonificación sísmica de la ciudad.

\section{Referencias Bibliográficas}

Álvarez-Icaza, Luis. (2014). Identificación de Rigidez En Los Entrepisos de Un Edificios Mediante Tiempos de Viaje de Onda. Congreso Nacional de Control Automático.

Ampuero, Alejandro. \& Van Sint, M. (2004). "Velocidades de Onda Medidas En Santiago Con El Ensayo de Refracción Sísmica." Geophysical Journal International, Doi: 182, 1, 355-367.

Bermeo Moyano, H. V. (2017). Determinación de Espectros de Respuesta y Espectros de

Diseño Elásticos e Inelásticos Para La Ciudad de Cuenca. 
Chávez, L. L. (2014). Efectos de Sitio Para Ingenieros Geotécnicos, Estudio Del Valle Parkway. Obras y Proyectos (16):6-30.

Ilori, A. O. (2019). Extracting Some soil parameters and estimating elastic settlements from direct shear box data for a granular c-empty set soil. SN APPLIED SCIENCES 1(10). Doi: 10.1007/s42452-019-1347-x.

Jiménez, J., Cabrera, J., Sánchez, J. \& Avilas, A. (2018). Vulnerabilidad sísmica del patrimonio edificado del Centro Histórico de la Ciudad de Cuenca: Lineamientos generales y avances del proyecto. MSKN MASKANA 9(1):59-78. ISSN: 13906143.doi.org/10.18537/mskn.09.01.0. Doi: doi.org/10.18537/mskn.09.01.07.

Mohammed, M. A., Abudeif, A. M. \& Abd, A. K. (2020). Engineering Geotechnical Evaluation of Soil for Foundation Purposes Using Shallow Seismic Refraction and MASW in 15th Mayo, Egypt. Journal of African Earth Sciences 162. Doi: 10.1016/j.jafrearsci.2019.103721.

Negreros, Ramiro. \& Gonzales, Alfonso. (2018). Universidad de San Carlos de Guatemala Facultad de Ingeniería Escuela de Ingeniería Civil.

Pickering, D. J. (1970). Anisotropic Elastic Parameters for Soil. Geotechnique 20(3):27176. Doi: 10.1680/geot.1970.20.3.271.

Taipe Acosta, L. M. (2013). Análisis de las ecuaciones de predicción de movimientos de suelo para el ecuador utilizando datos registrados durante el periodo 2000-2011 en las estaciones, sísmicas de banda ancha y acelerógrafos. Http://Bibdigital.Epn.Edu.Ec/Handle/15000/6447

Vanegas Camero, M. J. (2020). Modelación Experimental y Analítica Del Comportamiento Dinámico de Un Suelo Blando.

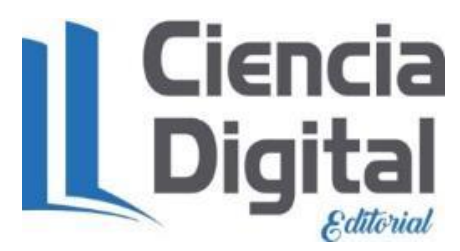




\section{Conciencia}

El artículo que se publica es de exclusiva responsabilidad de los autores y no necesariamente reflejan el pensamiento de la Revista Conciencia Digital.

\section{LCiencia}

El artículo queda en propiedad de la revista y, por tanto, su publicación parcial y/o total en otro medio tiene que ser autorizado por el director de la Revista Conciencia Digital.
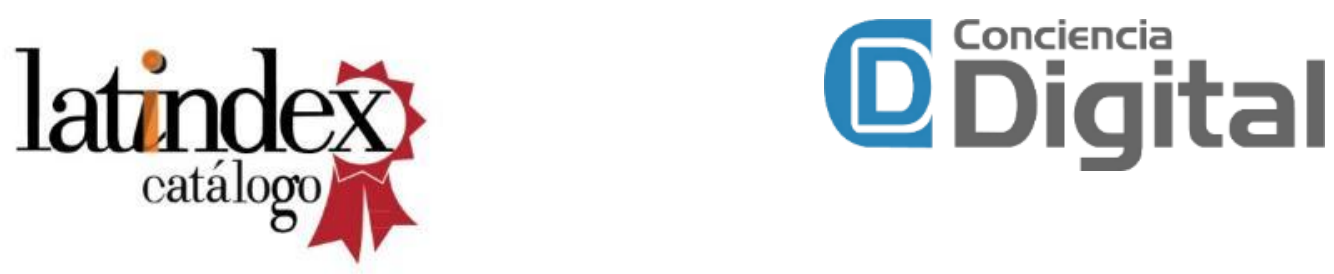

Indexaciones

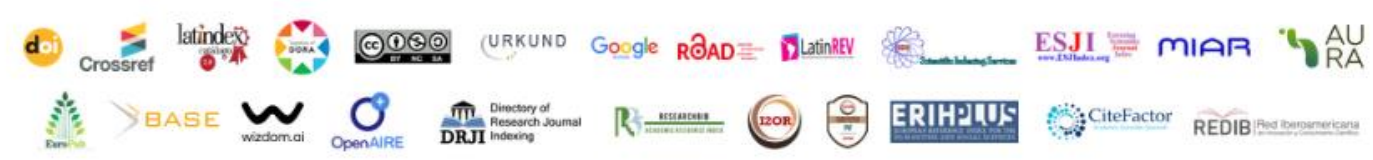

